\title{
Hamiltonian formulation of induced gravity in two dimensions
}

\author{
C. G. Torre \\ Center for Space Science Research, Space Research Institute, Florida Institute of Technology, Melbourne, Florida 32901-2715
}

(Received 22 May 1989)

\begin{abstract}
A Hamiltonian formulation of the theory of induced gravity in two dimensions is constructed. This formulation differs from previous efforts in that the formalism is covariant under all relevant transformation groups. In particular, spatial diffeomorphism covariance and/or invariance is manifest throughout; the phase space carries a representation of the Lie algebra of the spacetime diffeomorphism group; the group of conformal isometries is projectively represented on the phase space as a symmetry group. The key ingredient that allows covariance with respect to the above groups is the enlargement of the gravitational phase space by the inclusion of the cotangent bundle over the space of embeddings of a Cauchy surface into the spacetime.
\end{abstract}

\section{INTRODUCTION}

Interest in two-dimensional gravity has waxed once again due to recent work of Polyakov and others. ${ }^{1}$ Originally found in the context of string theory, ${ }^{2}$ this nonlocal theory of gravity also has applications in conformal field theory, statistical mechanics and, when quantized, as a model for $3+1$ quantum gravity. In the latter application especially, the Hamiltonian formulation of the theory is most useful since it allows one to model various proposals in quantum cosmology.

Hamiltonian formulations of induced gravity in two dimensions have come from Teitelboim ${ }^{3}$ and Marnelius. ${ }^{4}$ In Ref. 3, the two-metric is split into its conformally invariant and conformally dependent parts. The nonlocal gravitational action, which is induced by quantum matter, correspondingly splits into a conformally invariant and conformally dependent piece, neither of which is separately a diffeomorphism-invariant functional. The conformally dependent part of the action functional is local and, while coordinate dependent, by itself yields the correct covariant equation of motion provided one holds fixed the conformally invariant part of the metric.

In Ref. 4, an alternative, local action is constructed with the help of an auxiliary field. This alternative action has the virtue of being both local and diffeomorphism invariant. However, in order to eliminate the auxiliary field, the spacetime foliation is fixed to be adapted to a conformal coordinate system and the resulting Hamiltonian formalism is noncovariant in much the same way as in Ref. 3. One may summarize the situation by saying that in Teitelboim's approach the foliation is arbitrary but the action, and hence the Hamiltonian, is noncovariant, while in Marnelius' approach the action is invariant, but the foliation is fixed and the Hamiltonian is, at the end of the day, noncovariant.

In what follows we will blend these two approaches so as to obtain a Hamiltonian formulation of twodimensional gravity which behaves properly under all relevant transformation groups. In particular, we have in mind the following groups and/or Lie algebras: the spacetime diffeomorphism group $\operatorname{Diff}(M)$ and its Lie algebra $\operatorname{diff}(M)$; the group of diffeomorphisms of the spacelike (equal-time) slices $\operatorname{Diff}\left(S^{1}\right)$ and its Lie algebra $\operatorname{diff}\left(S^{1}\right)$; the group $\operatorname{Conf}(M, g)$ of conformal isometries, i.e., the subgroup of the diffeomorphism group which rescales the metric $g$ by a function, along with its Lie algebra $\operatorname{conf}(M, g)$. The key to keeping track of all these groups is the introduction of, as dynamical variables, the space of embeddings of Cauchy surfaces into the spacetime.

An outline of the paper is as follows. In Sec. II we summarize the properties of the nonlocal action. Because of the conformal simplicity of two dimensions, we show that two variational principles are possible, differing by whether one treats the conformal structure of $M$ as fixed or variable. The local action of Ref. 4 is described in Sec. III. Here again two variational principles can be used. Our central results are contained in Sec. IV. There we construct the Hamiltonian formulation of both variational principles and, after "parametrizing" the theory, show how they ultimately yield the same formalism. In Sec. V, we discuss the status of the three transformation groups that we have described. We have included two appendixes. The first demonstrates the canonical equivalence of two-dimensional gravity with the Hamiltonian formulation of embedding deformations in two dimensions. The second appendix provides some technical detail necessary for computing the symplectic structure on the gravitational phase space.

Throughout, latin indices are abstract indices for tensors in two dimensions, greek indices represent components in a coordinate basis which, unless otherwise specified, is arbitrary.

\section{NONLOCAL ACTION}

The form of the gravitational action is controlled completely by the conformal anomaly and the requirement of diffeomorphism invariance. It is given $b^{2}$

$$
\begin{aligned}
S[g]= & \frac{\alpha}{2} \int_{M \times M} \sqrt{-g(x)} R(x) G\left(x, x^{\prime}\right) \sqrt{-g\left(x^{\prime}\right)} R\left(x^{\prime}\right) \\
& +\lambda \int_{M} \sqrt{-g} .
\end{aligned}
$$


In the above, $M=R \times S^{1}$ is the two-dimensional spacetime manifold with Lorentzian metric $g_{a b}, R$ is the curvature scalar of $g$, and $G\left(x, x^{\prime}\right)$ is a Green's function satisfying

$$
g^{a b} \nabla_{a} \nabla_{b} G\left(x, x^{\prime}\right)=\frac{1}{\sqrt{-g}} \delta\left(x, x^{\prime}\right) .
$$

The "coupling constant" $\alpha$ depends on the species of quantum matter that has generated the action. For a single minimally coupled massless scalar field $\alpha=-\hbar / 48 \pi$. Henceforth we will choose units such that $\alpha=-1$. The parameter $\lambda$ is analogous to a cosmological constant and is freely variable. For simplicity we will set it to zero, although virtually all of the considerations of this paper hold when $\lambda$ is nonzero.
By a diffeomorphism, it is always possible to put the metric into the form

$$
g_{a b}=e^{\phi} f_{a b},
$$

where $f_{a b}$ is a flat metric on $M$. With this choice of metric, the action is local and equivalent to the action for a free scalar field $\phi$. However, to construct a Hamiltonian formalism meeting the requirements outlined in Sec. I, we will need a local action that yields the correct equations without the choice (2.3).

While the action (2.1) is nonlocal, the equations of motion obtained by varying it are essentially local. Thus the functional derivative of (2.1) with respect to the metric is given by

$$
\begin{aligned}
T_{a b}:=-\frac{2}{\sqrt{-g}} \frac{\delta S}{\delta g^{a b}} & \\
=g_{a b} R-\left(\delta_{a b}^{(c d)}-\frac{1}{2} g_{a b} g^{c d}\right) & 2 \nabla_{c} \nabla_{d} \int_{M} d x^{\prime} \sqrt{-g\left(x^{\prime}\right)} R\left(x^{\prime}\right) G\left(x, x^{\prime}\right) \\
& \left.-\int_{M} d x^{\prime} \sqrt{-g\left(x^{\prime}\right)} R\left(x^{\prime}\right) \partial_{c} G\left(x^{\prime}, x\right) \int_{M} d x^{\prime \prime} \sqrt{-g\left(x^{\prime \prime}\right)} R\left(x^{\prime \prime}\right) \partial_{d} G\left(x, x^{\prime \prime}\right)\right]
\end{aligned}
$$

where we have split the functional derivative into its pure-trace and trace-free components. The trace of $T_{a b}$ yields twice the curvature scalar as required by the conformal anomaly. Thus one of the equations of motion is simply

$$
R=0 \text {. }
$$

Equation (2.5) is in fact the only independent equation of motion because the trace-free part of (2.4) vanishes identically when $(2.5)$ holds. That $(2.5)$ is the only independent field equation is, of course, due to the fact that, once $R=0$, there are no other relevant geometric restrictions that can be placed on the two-dimensional spacetime.

The space of solutions to (2.5) is equivalent to the space of solutions to the scalar wave equation on $M$. To see this, choose conformal coordinates $x^{\alpha}$ in which the metric components are in the form (2.3); then (2.5) is equivalent to

$$
\frac{1}{\sqrt{-f}} \partial_{\alpha}\left(f^{\alpha \beta} \sqrt{-f} \partial_{\beta} \phi\right)=0 .
$$

Solutions to (2.6) are, however, "pure gauge" in the sense that one can always find a conformal isometry, which is the residual coordinate freedom left after imposing (2.3), such that $\phi=0$. In other words, if $R=0$ there will exist coordinates in which $g_{\alpha \beta}=f_{\alpha \beta}$.

The redundant field equations, contained in the tracefree part of $T_{a b}$, come from varying the conformally invariant part of $g_{a b}$. We therefore have the option of using a simpler variational principle: vary only the conformally dependent part of the metric. Then the only equation obtained is (2.5), and the equations of motion have the same content in either variational principle. ${ }^{5}$ By holding fixed part of the metric, we break diffeomorphism invariance; we will show how this can be restored when we pass to the Hamiltonian formulation. Having, for the time being, broken diffeomorphism invariance by working in a fixed conformal equivalence class of metrics, we can define an effective energy-momentum tensor for the gravitational field $\phi$. To do this, fix once and for all a flat metric $f_{a b}$ and define the metric via (2.3). The curvature scalar is now a fixed functional of the field $\phi$ :

$$
R=-e^{-\phi} f^{a b} \nabla_{a} \nabla_{b} \phi,
$$

where

$$
\nabla_{a} f_{b c}=0 \text {. }
$$

The functional derivative (2.4) can then be written

$$
\begin{aligned}
t_{a b}= & -\partial_{a} \phi \partial_{b} \phi+\frac{1}{2} f_{a b} f^{c d} \partial_{c} \phi \partial_{d} \phi \\
& -2 f_{a b} f^{c d} \nabla_{c} \nabla_{d} \phi+2 \nabla_{a} \nabla_{b} \phi .
\end{aligned}
$$

We emphasize that it was necessary to break diffeomorphism invariance to obtain this result. The effective energy-momentum tensor in $(2.8)$ is not equal to that of (2.4), the latter being trivial as is usual in generally covariant theories. Thus, when $R=0$, the trace-free part of $T_{a b}$ vanishes while the trace-free part of $t_{a b}$ does not vanish unless we choose the solution (i.e., via boundary conditions) $\phi=0$. It is not necessary to demand that the trace-free part of (2.8) vanish either since it is not an equation of motion of the restricted variational principle. Nevertheless, as mentioned above, when we factor the space of solutions of $R=0$ by the residual gauge group (conformal isometries) $\phi$ and hence $t_{a b}$ are equivalent to zero.

As we shall see, $t_{a b}$ in (2.8) does indeed play the role of an energy-momentum tensor in the Hamiltonian formulation. In this context, notice that the terms quadratic in $\phi$ have the wrong sign for a physical field as is also the case 
for the conformal degree of freedom in $(3+1)$ dimensional gravity. To construct the Hamiltonian formalism, we should start from a local action functional. Moreover this functional must respect the diffeomorphism invariance of the theory. Such an action was found by Marnelius and is the subject of the next section.

\section{LOCAL ACTION}

We begin with the action of Ref. $4:^{6}$

$S[g, \psi]=-\int_{M} \sqrt{-g}\left(\frac{1}{2} g^{a b} \psi_{, a} \psi_{, b}+R \psi\right)-2 \oint_{\mathrm{\partial} M} \sqrt{\gamma} K \psi$.

Here $\psi$ is a scalar field, $\gamma$ is the induced metric on the boundary of $M$ (assumed spacelike), $K$ is the mean extrinsic curvature of $\partial M$. As in (3+1)-dimensional gravity, the boundary term serves to eliminate second time derivatives of the metric from the action which are contained in $R$.

Following Sec. II, there are two variational principles available; they differ by whether or not one chooses to vary the conformally invariant part of the metric. In any case, the relevant functional derivatives are

$$
\begin{aligned}
& \frac{\delta S}{\delta \psi}=\sqrt{-g}\left(g^{a c} \nabla_{a} \nabla_{c} \psi-R\right), \\
& -\frac{2}{\sqrt{-g}} \frac{\delta S}{\delta g^{a b}}=\left(\psi_{, a} \psi_{, b}-\frac{1}{2} g_{a b} g^{c d} \psi_{, c} \psi_{, d}\right. \\
& \left.+2 g_{a b} g^{c d} \nabla_{c} \nabla_{d} \psi-2 \nabla_{a} \nabla_{b} \psi\right) .
\end{aligned}
$$

In (3.2) and (3.3) $\nabla$ is the derivative operator compatible with the metric $g$. Setting (3.2) to zero is equivalent to finding a conformal factor, $e^{\psi}$, which gives a rescaled metric $e^{\psi} g_{a b}$ with vanishing curvature. Hence if we solve (3.2) via

$$
\psi(x)=\int_{M} d x^{\prime} \sqrt{-g\left(x^{\prime}\right)} G\left(x, x^{\prime}\right) R\left(x^{\prime}\right)
$$

then (3.3) coincides with (2.4). Notice that (3.4) fixes $\psi$ purely in terms of geometry; all source-free waves are omitted. Thus there are no dynamical degrees of freedom for $\psi$ in (3.4). Using (3.4) and setting to zero the trace of (3.3) as field equations, we recover the results of Sec. II. In particular, if we hold fixed the conformal structure of $M$, we have available the local, effective energymomentum tensor (2.8), the scale factor satisfies (2.6), and there are no degrees of freedom left after factoring the space of solutions by $\operatorname{Conf}(M, g)$.

If the full metric is varied, then both (3.2) and (3.3) vanish as field equations. The traces of (3.3) and (3.2) together imply

$$
g^{a c} \nabla_{a} \nabla_{c} \psi=R=0
$$

and we have the trace-free part of (3.3) set to zero also. As we shall see, these latter two equations play the role of constraints and serve to eliminate all degrees of freedom from the theory. This is consistent with the results of Sec. II. Thus, irrespective of the choice of variational principle, the action (3.1) gives a local formulation of the theory based on (2.1). In fact, within the confines of the Hamiltonian formulation associated with (3.1), we shall show that the two variational principles are really two sides of the same coin. The relation between them will be the same as that between eliminating gauge redundancy by either fixing a gauge or working on the space of gauge orbits.

\section{HAMILTONIAN FORMULATION}

We will break this section into four parts. The first defines the phase space for the theory, the second and third parts treat the two variational principles separately, the fourth part relates them.

\section{A. Phase space}

Given a local action, there are many equivalent routes to the Hamiltonian formalism. To reach the desired goal of a completely covariant formalism, it will be most useful to proceed always keeping the foliation of $M$ arbitrary and the role of the embedded spacelike slices manifest.

Thus we begin by introducing a foliation $X$ of $M$ :

$$
X: R \times S^{1} \rightarrow M .
$$

In local coordinates $x^{\alpha}, \sigma, \tau$ on $M, S^{1}, R$, respectively, (4.1) reads

$$
x^{\alpha}=X^{\alpha}(\sigma, \tau) \text {. }
$$

The map (4.1) or (4.2) describes a one-parameter family of embeddings of a circle into $\boldsymbol{M}$. Embeddings which are infinitesimally close with respect to the parameter $\tau$ are related by a deformation along the vector $N^{\alpha}$, where

$$
N^{\alpha}:=\dot{X}^{\alpha} \equiv \frac{\partial X^{\alpha}}{\partial \tau} \text {. }
$$

$N^{\alpha}$ will be referred to as a "deformation vector."

As usual, the spacetime metric is given a $1+1$ decomposition via

$$
g_{a b} \rightarrow\left(N, N^{1}, \gamma\right)
$$

where

$$
\begin{aligned}
& \gamma=X^{\alpha}{ }_{, 1} X^{\beta}{ }_{, 1} g_{\alpha \beta}, \\
& N=-\gamma^{-1 / 2} n_{\alpha} N^{\alpha}, \\
& N^{1}=\gamma^{-1} g_{\alpha \beta} X^{\alpha}{ }_{, 1} N^{\beta},
\end{aligned}
$$

we use the notation $X^{\alpha}{ }_{1}=\partial_{\sigma} X^{\alpha}$. In the above $\gamma>0$ is the induced one-metric on the circles of constant $\tau ; N$ is the lapse density of weight minus 1 (Ref. 7), obtained by projecting the deformation vector along the unit normal $n_{\alpha}$ to a given embedding; $N^{1}$ is the shift vector obtained by projecting $N^{\alpha}$ along the tangent to the embedding. These latter two, essentially kinematic, functions represent the conformally invariant part of the spacetime metric, while the scale of $g_{a b}$ is represented by the onemetric $\gamma$.

The basic dynamical variables will be the field $\psi$ on a given slice along with the induced one-metric on that slice. Depending on the choice of variational principle, 
the lapse and shift will enter either as externally prescribed functions (conformal structure held fixed), or as freely variable Lagrange multipliers enforcing constraints (conformal structure varied). To obtain the canonical momenta conjugate to $\psi$ and $\gamma$, the Lagrangian density is pulled back by the foliation from $M$ to $R \times S^{1}$. The pullback of the first term in (3.1) is standard. To pull back the curvature scalar it is useful to use the identity (in two dimensions only)

$$
R=-2 \nabla_{a}\left(n^{a} K+n^{b} \nabla_{b} n^{a}\right),
$$

where $K$ is the mean extrinsic curvature of the time slices. Isolating velocity terms (derivatives with respect to $\tau$ ), and defining the momenta, the action (3.1) can be written as a functional on curves in the phase space $\Gamma^{\prime}$, which is the cotangent bundle over the product space of one-metrics and functions on the circle:

$$
S\left[\gamma, \Pi_{\gamma} ; \psi, \Pi_{\psi} ; N, N^{1}\right]=\int_{R \times S^{1}}\left(\Pi_{\gamma} \dot{\gamma}+\Pi_{\psi} \dot{\psi}-N h-N^{1} h_{1}\right),
$$

where

$$
h=-\frac{1}{2}\left(\gamma \Pi_{\gamma}\right)^{2}+\gamma \Pi_{\gamma} \Pi_{\psi}+\frac{1}{2}\left(\psi_{, 1}\right)^{2}-2 \gamma^{1 / 2} \partial_{1}\left(\gamma^{-1 / 2} \psi_{, 1}\right),
$$

$h_{1}=\Pi_{\gamma} \gamma_{, 1}-2 \partial_{1}\left(\gamma \Pi_{\gamma}\right)+\Pi_{\psi} \psi_{, 1}$.

The functions $h$ and $h_{1}$ are respectively the (weight-2) energy and momentum densities measured by observers moving along the integral curves of $n^{a}$.

Varying $S$ with respect to $\Pi_{\gamma}$ and $\Pi_{\psi}$ yields the definitions of the momenta. Together with these definitions, the equations coming from varying $\psi$ and $\gamma$ are equivalent to (3.5). Introducing the fundamental Poisson brackets

$$
\left[\gamma(\sigma), \Pi_{\gamma}\left(\sigma^{\prime}\right)\right]=\delta\left(\sigma, \sigma^{\prime}\right)=\left[\psi(\sigma), \Pi_{\psi}\left(\sigma^{\prime}\right)\right],
$$

the dynamical evolution (3.5) is generated by taking Poisson brackets with the Hamiltonian

$$
h(\mathbf{N}):=\int_{S^{1}}\left(N h+N^{1} h_{1}\right) .
$$

\section{B. Variable conformal structure}

Here we treat the lapse and shift as freely variable. This leads to the constraints

$$
h=0=h_{1} \text {. }
$$

As these equations come from varying the conformally invariant part of the metric, they correspond to the vanishing of the trace-free part of (3.3).

The constraints (4.13) reflect the underlying diffeomorphism invariance of theory and form, via Poisson brackets, the $1+1$ hypersurface deformation algebra. Thus define

$$
\begin{aligned}
& h_{( \pm)}:=\frac{1}{2}\left(h \pm h_{1}\right), \\
& h_{( \pm)}(N)=\int_{S^{1}} N h_{( \pm)},
\end{aligned}
$$

where $N$ is an arbitrary vector on $S^{1}$. Then we have the following nonvanishing Poisson brackets among the constraints:

$$
\left[h_{( \pm)}(N), h_{( \pm)}(M)\right]= \pm h_{( \pm)}\left(L_{N} M\right)
$$

where $L_{N}$ denotes the Lie derivative along $N$. As usual in $1+1$ dimensions, the constraint algebra consists of two copies of $\operatorname{diff}\left(S^{1}\right)$; i.e., the algebra is isomorphic to $\operatorname{conf}(M, g)$.

The closure of the algebra (4.16) indicates that the constraints are "first class." The dimensionality of the true, dynamical phase space is thus reduced by four from the initial choice [two from the constraints, two from factoring out the gauge group generated by (4.15)]. This leaves no field degrees of freedom, which is consistent with earlier reasoning. Notice that a generally covariant theory which is "pure gauge" can be considered to be a theory of embeddings. ${ }^{8}$ In Appendix A we demonstrate this explicitly for the theory based on the action (3.1).

The Hamiltonian formalism we have just developed is adequate for describing two-dimensional gravity. But it is somewhat unsatisfactory to have the auxiliary field $\psi$ present in the final form of the theory as it has no a priori geometric meaning. While $\psi$, like $\gamma$, is pure gauge, it is difficult to factor $\psi$ from the phase space while retaining $\gamma$ since they are coupled via (4.13). At any rate, working on a space of orbits is usually two unwieldy or too implicit. A more useful alternative is to work on a gauge slice that eliminates $\psi$. We will actually do this in the next subsection, but we will not interpret the results as such until the final subsection.

\section{Fixed conformal structure}

Using this variational principle amounts to treating $N$, $N^{1}$ in (4.8) as fixed, externally prescribed functions; the constraints (4.13) are not imposed. The equations of motion for the one-metric and auxiliary field still hold; they are equivalent to (3.5). Because the lapse and shift are fixed, the action (4.8) is no longer a diffeomorphism invariant functional. As one of our goals is a $\operatorname{diff}(\boldsymbol{M})$ covariant formalism, we will need to reestablish this symmetry. We do this not by varying the lapse and shift directly, but instead by adjoining the embeddings (and their conjugate momenta) to the phase space. This is a standard trick in mechanics, ${ }^{9}$ where it amounts to adding the time to the phase space, and is known as "parametrization."

To parametrize the theory, we exploit the fact that the conformally invariant part of the metric, i.e., $N, N^{1}$, is being held fixed. Thus, without loss of generality we can choose the two-metric to be of the form (2.3) for some fixed choice of the flat metric. In the Hamiltonian picture, varying $\phi$ is equivalent to varying $\gamma$ because there is the relation

$$
\gamma=e^{\phi} f
$$

where $f$ is the metric induced on the circle by the flat metric on $M$ :

$$
f=f_{\alpha \beta}[X] X^{\alpha}{ }_{, 1} X^{\beta}, 1 .
$$


Unlike $\gamma, f$ is a fixed, known functional of the embeddings (time) via (4.18). The lapse and shift, being conformally invariant, can be expressed solely in terms of the foliation $X$ and $f_{a b}$ :

$$
\begin{aligned}
N & =-f^{-1 / 2} m_{\alpha} \dot{X}^{\alpha}, \\
N^{1} & =f^{-1} f_{\alpha \gamma} X^{\alpha}{ }_{, 1} \dot{X}^{\gamma} \\
& \equiv X_{\gamma}^{1} \dot{X}^{\gamma} .
\end{aligned}
$$

In (4.19) $m_{\alpha}$ is the covector which is orthogonal to the slice (a conformally invariant notion) and normalized with respect to $f_{a b}$.

We now add the embeddings to the phase space as follows. Rewrite the action (4.8) as

$S\left[\gamma, \Pi_{\gamma} ; \psi, \Pi_{\psi}\right]=\int_{R \times S^{1}}\left(\Pi_{\gamma} \dot{\gamma}+\Pi_{\psi} \dot{\psi}-\dot{X}^{\alpha} h_{\alpha}\right)$,

where

$$
h_{\alpha}=-m_{\alpha} f^{-1 / 2} h+X_{\alpha}^{1} h_{1} .
$$

The embedding velocities (deformation vectors) $\dot{X}^{\alpha}$ only appear in the action as the coefficient of $h_{\alpha}$. Thus, if we treat $X^{\alpha}(\sigma)$ as a dynamical variable, the definition of the corresponding momentum $P_{\alpha}$ is a constraint:

$$
P_{\alpha}+h_{\alpha}=0 \text {. }
$$

The appearance of a new pair of constraints simply reflects the fact that the embeddings are not truly dynamical; they represent time. Momenta conjugate to time (space) are associated with energy (momentum) variables. This is the content of (4.23).

The action functional for the embedding-extended phase space, which we shall denote $\Gamma^{\prime \prime}$, takes the form

$$
\begin{aligned}
& S\left[\gamma, \mathrm{II}_{\gamma} ; \psi, \Pi_{\psi} ; X^{\alpha}, P_{\alpha} ; N^{\alpha}\right] \\
& \quad=\int_{R \times S^{1}}\left(\Pi_{\gamma} \dot{\gamma}+\Pi_{\psi} \dot{\psi}+P_{\alpha} \dot{X}^{\alpha}-N^{\alpha} H_{\alpha}\right),
\end{aligned}
$$

where the constraints

$$
H_{\alpha}:=P_{\alpha}+h_{\alpha} \approx 0,
$$

are imposed via the multipliers $N^{\alpha}$. Varying $S$ in (4.24) with respect to all of its arguments, we obtain a system of equations equivalent to those coming from (4.8) (when $N$, $N^{1}$ are held fixed). Using the Poisson brackets

$$
\left[X^{\alpha}(\sigma), P_{\beta}\left(\sigma^{\prime}\right)\right]=\delta_{\beta}^{\alpha} \delta\left(\sigma, \sigma^{\prime}\right),
$$

the equations of motion are generated by the Hamiltonian

$$
H(\mathbf{N})=\int_{S^{1}} N^{\alpha} H_{\alpha} .
$$

In particular, we have

$$
\dot{X}^{\alpha}=\left[X^{\alpha}, H(\mathbf{N})\right]=N^{\alpha} ;
$$

thus the multipliers $N^{\alpha}$ enforcing the constraints (4.25) represent a deformation vector. The equations

$$
\dot{P}_{\alpha}=\left[P_{\alpha}, H(\mathrm{~N})\right]
$$

ensure that the constraints are preserved in time. As the
Hamiltonian (4.27) is completely built out of the constraints, this implies they are first class. In fact, direct calculation reveals that the constraint algebra is Abelian:

$$
[H(\mathrm{~N}), H(\mathrm{M})]=0 \quad \forall N^{\alpha}, M^{\alpha}
$$

(for a general discussion, see Ref. 10).

As shown in Ref. 11 (see also Refs. 12 and 13), the relation (4.30) guarantees that $\Gamma^{\prime \prime}$ carries a representation of $\operatorname{diff}(M)$. The $\operatorname{diff}(M)$ representatives are simply the Hamiltonians (4.27), where the smearing fields are chosen to be spacetime vector fields restricted to an embedding:

$$
H(\mathbf{V}):=\int_{S^{1}} V^{\alpha}[X(\sigma)] H_{\alpha} .
$$

Because the smearing fields now depend on the embeddings, we have

$$
[H(\mathrm{~V}), H(\mathrm{~W})]=H(-[\mathrm{V}, \mathrm{W}]),
$$

where the brackets on the right-hand side of (4.32) is the commutator of vector fields. Thus $H(\mathrm{~V})$ provides a homomorphism from $\operatorname{diff}(M)$ into the Poisson algebra of functions on $\Gamma^{\prime \prime}$. It is the existence of the functions (4.31) satisfying (4.32) that indicates the general covariance of this formulation of the theory.

Having reestablished diffeomorphism invariance, we are now in a position to put the Hamiltonian theory into final form by eliminating the auxiliary field $\psi$. The strategy is to use (3.4), rewritten in phase-space language. As it is written, (3.4) is not very amenable to such a translation, but with the metric fixed to be conformal to a flat metric, (3.4) is equivalent to

$$
\psi=-\phi \text {. }
$$

To implement (4.33), perform a canonical transformation $^{14}$

$$
\left(\gamma, \Pi_{\gamma} ; \psi, \Pi_{\psi}: X^{\alpha}, P_{\alpha}\right) \rightarrow\left(\phi, P ; \psi_{0}, P_{\psi} ; \widetilde{X}^{\alpha}, \widetilde{P}_{\alpha}\right),
$$

where

$$
\begin{aligned}
& \gamma=e^{\phi} f[\widetilde{X}], \\
& \Pi_{\gamma}=e^{-\phi} f^{-1}[\widetilde{X}]\left(P+P_{\psi}\right), \\
& \psi=\psi_{0}-\phi \\
& \Pi_{\psi}=P_{\psi}, \\
& X^{\alpha}=\widetilde{X}^{\alpha}, \\
& P_{\alpha}=\widetilde{P}_{\alpha}+F_{\alpha}\left[\widetilde{X}, P, P_{\psi}\right] .
\end{aligned}
$$

The functions $F_{\alpha}$ will be defined shortly. There are a few properties of the transformation worth mentioning. First, it is easily verified to be canonical, e.g., by checking the invariance of the symplectic structure (using the definition of $F_{\alpha}$ below) or by constructing the generating functional. Notice that we have traded the one-metric for the scalar field $\phi$. The shifted auxiliary field $\psi_{0}$ is also a scalar and vanishes when (4.33) holds. Finally, observe that the canonical transformation is time dependent. The dependence on time enters via the one-metric $f[X(\sigma)]$ and extrinsic curvature (see below); it is mandatory due to our insistence on both manifest $\operatorname{Diff}\left(S^{1}\right)$ covariance 
and the use of an arbitrary foliation [needed for $\operatorname{diff}(M)$ covariance]. Recall that after performing a timedependent canonical transformation, the Hamiltonian must be supplemented by the time derivative of the generating functional. ${ }^{9}$ In the parametrized formalism this is automatically taken care of, i.e., in the term $F_{\alpha}$ in (4.39).

The new Hamiltonian takes the form (4.27) where (we now drop the tildes on the new embedding variables)

$$
H_{\alpha}=P_{\alpha}+h_{\alpha}+F_{\alpha} .
$$

It is instructive to give explicit expressions for $h_{\alpha}$ and $F_{\alpha}$ in two bases. First, we project them perpendicular and parallel to an embedded circle:

$$
\begin{aligned}
& h:=f^{1 / 2} m^{\alpha} h_{\alpha}=-\frac{1}{2}\left[P^{2}+\left(\phi_{, 1}\right)^{2}\right]+2 D_{1} D_{1} \phi \\
& \quad+\frac{1}{2}\left[P_{\psi}^{2}+\left(\psi_{0,1}\right)^{2}\right]-2 D_{1} D_{1} \psi_{0}, \\
& h_{1}:=X^{\alpha}{ }_{, 1} h_{\alpha}=P \phi_{, 1}-2 D_{1} P+P_{\psi} \psi_{0,1}-2 D_{1} P_{\psi}, \\
& F:=f^{1 / 2} m^{\alpha} F_{\alpha}=2 K_{1}\left(P_{\psi}+P\right), \\
& F_{1}:=X^{\alpha}{ }_{, 1} F_{\alpha}=2 D_{1}\left(P_{\psi}+P\right) .
\end{aligned}
$$

In the above we have denoted by $D_{1}$ the derivative operator on $S^{1}$ that is compatible with $f$,

$$
D_{1} f=0 \text {, }
$$

while $K_{1}$ is the mean extrinsic curvature of the embedding, defined with respect to $f_{a b}$ and rescaled with $f$ to be a scalar density of weight 1 (covector):

$$
K_{1}:=f^{1 / 2} K[f] \text {. }
$$

From the projected form of $h_{\alpha}$ it is clear that the contributions to the energy from the metric and auxiliary field are equal and opposite, the auxiliary field having the physical sign. Notice also that the canonical transformation has neatly decoupled the metric and auxiliary variables in (4.41)-(4.44).

It is also useful to see $h_{\alpha}$ and $F_{\alpha}$ expressed in a null coordinate system on $M$. Choose coordinates $x^{ \pm}$such that the line element corresponding to the metric $(2.3)$ is

$$
d s^{2}=-e^{\phi} d x+d x^{-}
$$

then

$$
\begin{gathered}
h_{ \pm}=\left(X_{, 1}^{ \pm}\right)^{-1}\left[\mp \frac{1}{4}\left(P \mp \phi_{, 1}\right)^{2}-D_{1}\left(P \mp \phi_{, 1}\right)\right. \\
\left. \pm \frac{1}{4}\left(P_{\psi} \pm \psi_{0,1}\right)^{2}-D_{1}\left(P_{\psi} \pm \psi_{0,1}\right)\right], \\
F_{ \pm}=\left(X_{, 1}^{ \pm}\right)^{-1( \pm)} D_{1}\left(P_{\psi}+P\right) .
\end{gathered}
$$

In (4.49) we have introduced a pair of derivative operators ${ }^{( \pm)} \mathbf{D}_{1}$. They act on covectors on the circle as

$$
{ }^{( \pm)} \mathbf{D}_{1} v_{1}=D_{1} v_{1} \pm K_{1} v_{1}
$$

with obvious generalizations to other tensors. ${ }^{15}$

The auxiliary field is eliminated by setting

$$
\psi_{0}=0, \quad P_{\psi}=-2 K_{1} \text {. }
$$

The restrictions (4.51) can be viewed as constraints on allowable initial data. Preservation of these constraints throughout the dynamical evolution generated by $H(\mathbf{N})$ leads to an identity equivalent to (4.7). Thus, restricting the extended phase space $\Gamma^{\prime \prime}$ to the subspace $\Gamma$ specified by $(4.51)$ is consistent with the dynamics of the theory. Notice that the constraints $(4.51)$ serve to eliminate propagating degrees of freedom for the auxiliary field.

Having chosen to work on the submanifold $\Gamma$, one must also work with the symplectic structure induced on $\Gamma$ from $\Gamma$ ", i.e., construct the "Dirac brackets." This is done in Appendix B where it is shown that the Dirac brackets of the remaining variables on $\Gamma,\left(\phi, P, X^{\alpha}, P_{\alpha}\right)$, are identical to their Poisson brackets. Thus it is permissible to eliminate $\psi_{0}$ and $P_{\psi}$ explicitly and work on $\Gamma$ using the original Poisson brackets for the surviving variables.

After eliminating the auxiliary field and its conjugate momentum, the Hamiltonian density (4.40)-(4.44), or (4.48) and (4.49), reduces to

$$
\mathbf{H}_{\alpha}=\boldsymbol{P}_{\alpha}+\mathbf{h}_{\alpha}+\mathbf{F}_{\alpha}
$$

where

$$
\begin{aligned}
& \mathbf{h}=-\frac{1}{2}\left[P^{2}+\left(\phi_{, 1}\right)^{2}\right]+2 D_{1} D_{1} \phi+2\left(K_{1}\right)^{2}, \\
& \mathbf{h}_{1}=P \phi_{, 1}-2 D_{1} P+4 D_{1} K_{1}, \\
& \mathbf{F}=2 K_{1}\left(P-2 K_{1}\right) \\
& \mathbf{F}_{1}=2 D_{1}\left(P-2 K_{1}\right),
\end{aligned}
$$

or

$$
\begin{gathered}
\mathbf{h}_{ \pm}=\left(X^{ \pm}{ }_{, 1}\right)^{-1}\left[\mp \frac{1}{4}\left(P \mp \phi_{, 1}\right)^{2}-D_{1}\left(P \mp \phi_{, 1}\right)\right. \\
\left. \pm\left(K_{1}\right)^{2}+2 D_{1} K_{1}\right] \\
\mathbf{F}_{ \pm}=\left(X^{ \pm}{ }_{, 1}\right)^{-1( \pm)} D_{1}\left(P-2 K_{1}\right) .
\end{gathered}
$$

The functions $h_{\alpha}$ have the interpretation of an effective energy-momentum flux for the gravitational field. It can be checked that these functions are related to the energy-momentum tensor (2.8) via

$$
\mathbf{h}_{\alpha}=f^{1 / 2} m^{\beta} t_{\alpha \beta} \text {. }
$$

Combining the expressions (4.52)-(4.56), we can write the super-Hamiltonian and supermomentum for the theory:

$$
\begin{aligned}
& \mathbf{H}:=f^{1 / 2} m^{\alpha} \mathbf{H}_{\alpha}= f^{1 / 2} m^{\alpha} P_{\alpha}-\frac{1}{2}\left[P^{2}+\left(\phi_{, 1}\right)^{2}\right] \\
&+2 D_{1} D_{1} \phi+2 K_{1}\left(P-K_{1}\right), \\
& \mathbf{H}_{1}:=X^{\alpha}{ }_{, 1} \mathbf{H}_{\alpha}=X^{\alpha}{ }_{, 1} P_{\alpha}+P \phi_{, 1} .
\end{aligned}
$$

The supermomentum is standard; it simply indicates that $\phi$ and $X^{\alpha}$ are scalar functions on the circle. The superHamiltonian is somewhat complicated; it is explicitly time dependent and, in particular, depends on the extrinsic curvature of the embedded slice. As we shall see in Sec. V, this time dependence is necessary for the spacetime diffeomorphism covariance of the theory.

The final form for the phase-space action functional on $\Gamma$ is 


$$
S\left[\phi, P ; X^{\alpha}, P_{\alpha} ; N^{\alpha}\right]=\int_{R \times S^{1}}\left(P \dot{\phi}+P_{\alpha} \dot{X}^{\alpha}-N^{\alpha} \mathbf{H}_{\alpha}\right)
$$

Varying $S$ in (4.62) with respect to all of its arguments leads to equations equivalent to (2.6) and hence (2.5). The constraints $\mathbf{H}_{\alpha}=0$, obtained by varying $N^{\alpha}$, still have an Abelian Poisson-brackets algebra, as can be checked by straightforward computation. Ultimately, the reason these constraints remain first class is simply that the constraint surface $\Gamma$ is preserved by the dynamical evolution generated by the Hamiltonian which, in turn, is built out of the constraints. The presence of these first-class constraints guarantees the elimination of $\left(X^{\alpha}, P_{\alpha}^{\cdot}\right)$ as dynamical degrees of freedom. As mentioned previously, we should also reduce the phase space by the action of the group $\operatorname{Conf}(M, g)$. We will return to the action of this group on the phase space in the next section.

\section{Relationship between Secs. IV B and IV C}

The action functional (4.62) is also appropriate for the Hamiltonian formulation of the variational principle of Sec. IV B provided one supplements the equations coming from varying (4.62) with the original Hamiltonian and momentum constraints (evaluated on $\Gamma$ )

$$
\mathbf{h}=0=\mathbf{h}_{1} \text {. }
$$

It can be checked that the time evolution generated by the Hamiltonian $\mathbf{H}(\mathbf{N})$ preserves these constraints in time. This is a consequence of the relation (4.59) and the fact that if

$$
f^{a c} \nabla_{a} \nabla_{c} \phi=0
$$

(here we use the connection compatible with $f$ ) then

$$
\nabla_{a} t^{a b}=0
$$

From the point of view of Sec. IV B, the constraints (4.51), which govern the elimination of the auxiliary field, represent a pair of gauge-fixing conditions. Consistent with this interpretation is the fact that the constraints (4.63) are second class. Thus, using $\mathbf{h}_{( \pm)}$smeared with vectors $N, M$ on the circle,

$\left[\mathbf{h}_{( \pm)}(N), \mathbf{h}_{( \pm)}(M)\right]= \pm h_{( \pm)}\left(L_{N} M\right)+{ }^{( \pm)} F(N, M) ，$

where

$$
{ }^{( \pm)} F(N, M)= \pm 2 \int_{S^{1}}\left({ }^{( \pm)} \mathbf{D}_{1}{ }^{( \pm)} \mathbf{D}_{1} N\right){ }^{( \pm)} \mathbf{D}_{1} M
$$

Closure of the constraint algebra is broken by the presence of the "anomalous" term $F$. This term is identical to the $\operatorname{Diff}\left(S^{1}\right)$-invariant Schwinger term in the quantum algebra of normal-ordered energy-momentum densities of a massless scalar field on a fixed cylindrical background. ${ }^{12}$ The presence of covariant derivatives in (4.67), rather than ordinary ones, is necessitated by the fact that the smearing fields $N, M$ must be vector fields on the circle.

The extended phase space of the parametrized Hamiltonian formalism thus allows the two variational principles of Secs. IV B and IV C to coalesce. More precisely, the two variational principles are simply alternative for- mulations of a trivial theory, differing only by whether the gauge degrees of freedom are frozen by (gauge-fixed) second-class constraints, (4.63), or by working on the space of orbits of a symmetry group $[\operatorname{Conf}(M, g)]$ which, as we shall see, is projectively represented on $\Gamma$.

\section{TRANSFORMATION GROUPS}

We are now able to evaluate the status of the three transformation groups listed in Sec. I. Using the equivalence between tensors and densities in one dimension, ${ }^{7}$ it is easy to verify that all elements of the Hamiltonian formalism are covariant/invariant under the spatial diffeomorphism group $\left[\operatorname{Diff}\left(S^{1}\right)\right]$. As for the spacetime diffeomorphism group $\operatorname{Diff}(M)$, we have seen how its Lie algebra is represented on the phase space $\Gamma^{\prime \prime}$. That $\operatorname{diff}(M)$ is also represented on $\Gamma$ is guaranteed by the relation

$$
\left[\mathbf{H}_{\alpha}(\sigma), \mathbf{H}_{\beta}\left(\sigma^{\prime}\right)\right]=0 .
$$

Equation (5.1) holds by virtue of the explicit time dependence of the super-Hamiltonian (4.60), which is needed to help cancel the "anomaly" generated by the energymomentum flux. Thus the functions

$$
\mathbf{H}(\mathbf{V})=\int_{S^{1}} V^{\alpha}[X(\sigma)] \mathbf{H}_{\alpha}
$$

provide a homomorphism from $\operatorname{diff}(M)$ into $C^{\infty}(\Gamma, R)$. Alternatively, the algebra of the functions (4.60), (4.61) is precisely the $1+1$ hypersurface deformation algebra (without center).

The spacetime diffeomorphism group enters the theory as a dynamical group: the functions (5.2) are the Hamiltonians of the theory. Similarly, the conformal group, $\operatorname{Conf}(M, g)$, can also be treated as a dynamical group by replacing $V^{\alpha}$ in (5.2) with a conformal Killing vector. ${ }^{12}$ As shown in Ref. 13, in two dimensions $\operatorname{Conf}(M, g)$ is just big enough to generate all dynamical evolutions. Rather than represent $\operatorname{Conf}(M, g)$ (or rather, its Lie algebra) as a subgroup of $\operatorname{Diff}(M)$, it is more interesting to study its status as a symmetry group for the theory. More precisely, is there an action of $\operatorname{Conf}(M, g)$ on solutions $(\phi(\sigma, \tau), P(\sigma, \tau))$ that leaves fixed the embeddings, while generating a new solution to the field equations? That the answer is in the affirmative can be seen by examining the effective energy-momentum tensor (2.8). We have already made note of the relations (4.64), (4.65). It is easy to see that if (4.64) holds, then we also have

$$
f_{a b} t^{a b}=0 \text {. }
$$

So, forming the covector

$$
j_{a}=v^{b} t_{a b},
$$

where $v^{a}$ is a conformal Killing vector,

$$
L_{v} f_{a b}=\Omega f_{a b}
$$

then, by virtue of (4.65) and (5.3), $j_{a}$ is divergence-free. Thus the quantities

$$
\mathbf{j}(\mathbf{v})=\int_{S^{1}} d \sigma f^{1 / 2} m^{a} j_{a},
$$


where the integral is over a spacelike slice of $M$, are constants of motion. However, the function (5.6) is nothing but the energy-momentum flux (4.59) smeared with a conformal Killing vector restricted to an embedding. Thus the functions on $\Gamma$,

$$
\mathbf{h}(\mathbf{v})=\int_{S^{1}} v^{\alpha}[X(\sigma)] \mathbf{h}_{\alpha},
$$

are constants of motion, i.e., have vanishing Poisson brackets with the Hamiltonian

$$
[\mathbf{h}(\mathbf{v}), \mathbf{H}(\mathbf{N})]=0 \quad \forall N^{\alpha} .
$$

To uncover the canonical transformation generated by (5.7), work in the null coordinates on $M$, then

$$
v^{ \pm}=v^{ \pm}\left(x^{ \pm}\right)
$$

and

$$
\begin{aligned}
\delta_{v} \phi:= & {[\phi, \mathbf{h}(\mathbf{v})] } \\
= & \frac{1}{2}\left(X^{+}{ }_{, 1}\right)^{-1} v^{+}\left[X^{+}\right]\left(-P+2 K_{1}+\phi_{, 1}\right) \\
& +\frac{1}{2}\left(X^{-}{ }_{, 1}\right)^{-1} v^{-}\left[X^{-}\right]\left(P-2 K_{1}+\phi_{, 1}\right) \\
& +v^{+}{ }_{,+}\left[X^{+}\right]+v^{-},-\left[X^{-}\right] .
\end{aligned}
$$

The portion of (5.9) containing the undifferentiated components of $v^{\alpha}$ is precisely the dynamical evolution generated by

$$
\mathbf{H}(\mathbf{v})=\int_{S^{1}} v^{\alpha} \mathbf{H}_{\alpha}
$$

and hence corresponds to the Lie derivative of $\phi$ along $v^{\alpha}$. Thus, generalizing to an arbitrary coordinate system, we have

$$
\delta_{v} \phi=L_{v} \phi+\nabla_{a} v^{a} .
$$

The transformation $(5.11)$ is easily recognized to be the natural change in a conformal factor due to a conformal isometry. The presence of the inhomogeneous term $\nabla_{a} v^{a}$ is responsible for the appearance of a center ${ }^{16}$ in the algebra of the associated canonical transformations:

$$
[\mathbf{h}(\mathbf{v}), \mathbf{h}(\mathbf{w})]=\mathbf{h}([\mathbf{v}, \mathbf{w}])+F(\mathbf{v}, \mathbf{w}),
$$

where

$$
\boldsymbol{F}(\mathbf{v}, \mathbf{w})={ }^{(+)} \boldsymbol{F}\left[\frac{v^{+}}{X^{+}, 1}, \frac{w^{+}}{X^{+}, 1}\right]+{ }^{(-)} F\left[\frac{v^{-}}{X^{-}, 1}, \frac{w^{-}}{X_{, 1}^{-}}\right],
$$

which follows directly from (4.66) and

$$
h_{ \pm}= \pm\left(X_{, 1}^{ \pm}\right)^{-1} h_{( \pm)}
$$

(see Ref. 12 for a coordinate-independent expression of $F)$.

$F(\mathbf{v}, \mathbf{w})$ can be verified to be a constant of motion, i.e., embedding independent, so $F$ represents a central extension of conf $(M, g)$. We conclude that, as is natural for a conformal factor, the conformal group is projectively represented as a symmetry group on $\Gamma$. Using this symmetry to reduce the phase space leads to a theory with no dynamical degrees of freedom.

\section{ACKNOWLEDGMENTS}

This work was stimulated by a discussion with I. Bakas. The author wishes to thank K. Kuchař for many useful discussions on all aspects of this work.

\section{APPENDIX A}

In the Hamiltonian description of a generally covariant system one typically encounters "pure gauge," or kinematic, variables that are signaled by the presence of constraints. In one form or another, these redundant variables represent time and, for field theories, space also. Thus, in the usual treatment of the relativistic point particle, there arises a constraint: the mass-shell condition. Correspondingly, there is a kinematic phase-space pair: the time in a given Minkowski frame and its conjugate momentum which, via the constraint, is given the meaning of energy as measured in that frame. A completely analogous situation arises for the relativistic string ${ }^{13}$ where now the kinematic variables represent spacelike embeddings and their conjugate momenta.

It is tempting to suppose that one can extract the "many-fingered time" canonical pairs from the phase space of any generally covariant system, e.g., general relativity, although in this theory one has never been completely successful. The simplicity of two dimensions does allow a successful extraction of the kinematic phasespace pairs for induced gravity based on the action (3.1). As we have seen, this theory has no (field) degrees of freedom so we expect that the theory should be completely kinematic, i.e., solely a theory of embeddings. ${ }^{8}$ Thus consider the transformation ${ }^{17}$

$$
\begin{aligned}
& \left(\gamma, \Pi_{\gamma} ; \psi, \Pi_{\psi}\right) \rightarrow\left(T, P_{T} ; S, P_{S}\right), \\
& \gamma^{-1} \gamma_{, 1}=-P_{S}+\partial_{1} \ln \left[\left(S_{, 1}\right)^{2}-\left(T_{, 1}\right)^{2}\right]+\frac{1}{2} T_{, 1}, \\
& \psi, 1=-T_{, 1}, \\
& \gamma \Pi_{\gamma}=-S_{, 1}, \\
& \Pi_{\psi}=-P_{T}-\frac{1}{2} S_{, 1}+\partial_{1} \ln \left(\frac{S_{, 1}+T_{, 1}}{S_{, 1}-T_{, 1}}\right) .
\end{aligned}
$$

If the spatial manifold is $R^{1}$ rather than $S^{1}$, this is a canonical transformation. We will deal with the complications of a "closed universe" below. Notice that the transformation is well defined only if

$$
\left(S_{, 1}\right)^{2}-\left(T_{, 1}\right)^{2}>0
$$

which implies

$$
\left(\gamma \Pi_{\gamma}\right)^{2}-\left(\psi_{, 1}\right)^{2}>0 \text {. }
$$

This inequality can be viewed as a restriction on the auxiliary field provided $\gamma \Pi_{\gamma} \neq 0$.

In terms of the new variables the functions (4.9) and (4.10) become

$$
\begin{aligned}
& h=T_{, 1} P_{S}+S_{, 1} P_{T}, \\
& h_{1}=T_{, 1} P_{T}+S_{, 1} P_{S} .
\end{aligned}
$$

These are precisely the super-Hamiltonian and super- 
momentum describing the kinematics of hypersurface deformations when the embeddings are registered in a conformal coordinate system. ${ }^{8,13}$

For spatial topology $S^{1}$ the transformation (A1)-(A4) is not canonical owing to the existence of a kernel for $\partial_{1}$. Thus, when we try to integrate the transformation, in particular (A1) and (A3), there is an unspecified integration constant in (A3) and a missing mode for $P_{S}$ in (A1). The origin of this difficulty in geometric terms is as follows. The function $S$ is to define a map between circles. As we must work in local coordinates, there must be a preferred point, i.e., where we must make an identification to produce the circle. However, there is no way to extract a preferred point from the phase-space data since, in general, such a point does not exist. If space is topologically $R^{1}$ this problem does not arise because the preferred point can be taken at infinity. A related feature of (A1)-(A4) is that there is no way to incorporate the constant mode of $\ln \gamma$ into the embedding variables. Thus, strictly speaking, one mode of the metric is dynamical. In the absence of matter fields, then, we are one mode short of identifying the phase space $\Gamma^{\prime}$ with the cotangent bundle over the space of embeddings. This missing mode, if it existed, would represent a choice of origin on the circle.

A remedy for this situation can be found in Ref. 13 . The idea is to supplement $\Gamma^{\prime}$ with one additional phase space pair, denoted $(q, p)$, which is rendered trivial by an extra constraint $p \approx 0$. This extra set of variables provides the gauge degrees of freedom necessary to describe the choice of origin. If gravity is coupled to matter, one can use a canonical pair from the matter sector to play the role of $(q, p)$. However, from the above geometric interpretation, one expects some difficulty in isolating suitable matter variables as they would have to provide a preferred choice of origin on a given slice.

\section{APPENDIX B}

We are interested in computing the symplectic structure induced on the submanifold $\Gamma \subset \Gamma^{\prime \prime}$, which is defined by the constraints (4.51). In general, given a symplectic submanifold $\Gamma$ of a phase space $\Gamma^{\prime \prime}$ specified by the vanishing of a set of functions,

$$
\chi_{\alpha}=0 \text {, }
$$

the Poisson brackets [, ] defined with respect to the induced symplectic structure on $\Gamma$ are defined in terms of the Poisson brackets $[,]^{\prime \prime}$ of $\Gamma^{\prime \prime}$ by

$$
[A, B]=[A, B]^{\prime \prime}-\left[A, \chi_{\alpha}\right]^{\prime \prime} D^{\alpha \beta}\left[\chi_{\beta}, B\right]^{\prime \prime},
$$

where $A, B \in C^{\infty}\left(\Gamma^{\prime \prime}, R\right)$, and

$$
D^{\alpha \beta}\left[\chi_{\beta}, \chi_{\gamma}\right]^{\prime \prime}=\delta_{\gamma}^{\alpha}=\left[\chi_{\gamma}, \chi_{\beta}\right]^{\prime \prime} D^{\beta \alpha}
$$

The brackets on $\Gamma$ are usually called the "Dirac brackets." 18

Computing the matrix $D^{\alpha \beta}$ for the constraints (4.51) with

$$
\chi_{1}=\psi_{0}, \quad \chi_{2}=P_{\psi}+2 K_{1}
$$

we find

$$
D^{11}=D^{22}=0, \quad D^{12}=-D^{21}=-\delta\left(\sigma, \sigma^{\prime}\right) .
$$

Using (B5) in (B2), it is straightforward to verify that the Dirac brackets for the remaining phase-space variables, i.e., the coordinates of $\Gamma$, are identical to their Poisson brackets. Thus, for $a, b, \in C^{\infty}(\Gamma, R)$,

$$
[a, b]=[a, b]^{\prime \prime} \text {. }
$$

${ }^{1}$ A. M. Polyakov, Mod. Phys. Lett. A 2, 893 (1987); V. G. Knizhnik, A. M. Polyakov, and A. B. Zamolodchikov, ibid. 3, 819 (1988); see also J. Polchinski, University of Texas Report No. UTTG-02-89, 1989 (unpublished), and references within.

${ }^{2}$ A. M. Polyakov, Phys. Lett. 101B, 207 (1981).

${ }^{3}$ C. Teitelboim, in Quantum Theory of Gravity, edited by S. M. Christensen (Adam Hilger, Bristol, 1984).

${ }^{4}$ R. Marnelius, Nucl. Phys. B211, 14 (1983).

${ }^{5}$ When coupling to matter is considered however, this equivalence no longer holds. Thus if we couple to conformally invariant matter (2.5) still follows from varying (2.1), but if we vary the full metric, the matter energy-momentum tensor is set to zero. In the context of string theory this is desirable, but for physical matter it is not; in this case the restricted variational principle (conformal structure fixed) seems most appropriate.

${ }^{6}$ If we make the rescaling $g_{a b}=e^{-\psi / 2} \bar{g}_{a b}$, then (3.1) becomes the action proposed by R. Jackiw in Quantum Theory of Gravity (Ref. 3).

${ }^{7}$ It is useful to recall that, on a one-dimensional manifold, all scalar densities of weight plus (minus) $w$ can also be con- sidered as covariant (contravariant) tensors of rank $w$.

${ }^{8}$ K. Kuchař, J. Math. Phys. 19, 390 (1978).

${ }^{9} \mathrm{C}$. Lanczos, The Variational Principles of Mechanics (University of Toronto Press, Toronto, 1970).

${ }^{10}$ K. Kuchař, J. Math. Phys. 17, 801 (1976).

${ }^{11}$ C. Isham and K. Kuchař, Ann. Phys. (N.Y.) 164, 288 (1985).

${ }^{12}$ K. Kuchař, Phys. Rev. D 39, 1579 (1989); 39, 2263 (1989).

${ }^{13}$ K. Kuchař and C. G. Torre, J. Math. Phys. 30, 1769 (1989); see also Proceedings of the Osgood Hill Conference on Conceptual Problems in Quantum Gravity, edited by A. Ashtekar and J. Stachel (Einstein Studies Series, Vol. 2) (Birkhauser, Boston, in press).

${ }^{14}$ This tranformation is a covariant generalization of the transformation used in Ref. 4.

${ }^{15}$ These derivative operators were first introduced by K. Kuchar in Ref. 12 and are the $(1+1)$-dimensional analogs of the derivative operators introduced into $3+1$ canonical gravity by A. Ashtekar, Phys. Rev. D 36, 1587 (1987).

${ }^{16}$ Note, that, in general, there is an $\operatorname{SL}(2, R) \otimes \operatorname{SL}(2, R)$ subgroup of $\operatorname{Conf}(M, g)$ which is represented without a two-cocycle. This subgroup corresponds to the conformal Killing vectors which set $F(\mathbf{v}, \mathbf{w})=0$. For the $S^{1}$ spatial sections we are con- 
sidering here, periodicity requirements will reduce this subgroup to $R \otimes R$.

${ }^{17}$ This canonical transformation was found during discusions with $\mathrm{K}$. Kuchař, and is based on a similar transformation in the canonical formulation of the dynamics of cylindrical gravitational waves; see K. Kuchař, Phys. Rev. D 4, 955 (1971).

${ }^{18}$ P. A. M. Dirac, Lectures on Quantum Mechanics (Belfer Graduate School of Science, Yeshiva University, New York, 1964). 